\title{
LA SATIRE SOCIALE DANS SORCELLERIE À BOUT PORTANT D'ACHILLE NGOYE
}

\author{
Dame KANE \\ Université Cheikh Anta Diop de Dakar, Sénégal \\ dame_4@hotmail.com \\ Article reçu le 6 avril 2017| révisé depuis le 25 avril 2017| accepté le 23 juin 2017
}

\begin{abstract}
RÉSUMÉ. Aux lendemains de la tragique guerre civile congolaise de 1998 à 2002 se terminant par la chute du dictateur Mobutu Sésé Séko, le Mal, tel une pieuvre à multiple tentacules, continuera à persister à la République démocratique du Congo, ce grand pays $\mathrm{d}^{\prime}$ Afrique centrale au sous-sol si riche en minerais de toutes sortes. Cette situation paradoxale poussera certains esprits révoltés comme Achille Ngoye dans Sorcellerie à bout portant à s'attaquer aux problèmes qui ont fini de mettre à genoux son pays. C'est donc une manière, pour lui, de lutter contre les effets désastreux du silence et de la résignation mais aussi de défendre son peuple meurtri par tant de violence. Cet article s'intéresse, ainsi, aux diverses manifestations du Mal dans ce roman négro-africain contemporain.
\end{abstract}

Mots-clés : analyse, criminalité, négro-africain, roman contemporain, satire sociale, sorcellerie.

\begin{abstract}
In the aftermath of the tragic Congolese civil war of 1998-2002, which ended with the fall of the dictator Mobutu Sese Seko, Evil, like an octopus with multiple tentacles, will continue to persist in the Democratic Republic of Congo, in the basement so rich in minerals of all kinds. This paradoxical situation will push some rebellious spirits like Achille Ngoye into Witchcraft at close range to tackle the problems that have ended up kneeling his country. It is thus a way for him to fight against the disastrous effects of silence and resignation but also to defend his people bruised by so much violence. This article is interested in the various manifestations of Evil in this contemporary Negro-African novel.
\end{abstract}

Keywords: analysis, criminality, negro-african, contemporary novel, social satire, witchcraft.

\section{INTRODUCTION}

Les faits sociaux occupent une place centrale dans plusieurs romans négroafricains. En Afrique comme partout ailleurs les sociétés ne sont pas seulement marquées par des réalités positives. Dans toutes les communautés à côté des «roses» on voit toujours dans une certaine mesure des «épines » debout comme des montagnes.
Certains auteurs abordent les pratiques sociales de manière sélective et exaltante du fait surtout qu'ils sont animés par un désir profond de ne montrer que le meilleur visage de leurs terres d'origines. Dans leurs écrits ils montrent une certaine harmonie sociale, la douceur de la vie, la solidarité africaine, le merveilleux, la culture, une Afrique qui sourit qui chante ou qui 
danse. Cependant d'autres semblent plutôt s'intéresser aux maux qui gangrènent leurs sociétés : dégradation des mœurs, alcoolisme, impunité, corruption, guerre... C'est le cas de beaucoup d'écrivains qui vont s'imposer depuis les Indépendances jusqu'à nos jours. Sony Labou-Tansi, Henri Lopes, Ahmadou Kourouma, William Sassine et tant d'autres romanciers de l'Afrique subsaharienne vont produire des textes dans lesquels la satire sociale y est omniprésente. Dans Sorcellerie à bout portant, Ngoye (1998) dresse un tableau sans complaisance de la société congolaise d'où il est issu.

Malgré la pluralité des recherches sur la thématique du mal dans la littérature négro-africaine francophone, il faut noter que cette œuvre de Ngoye qui doit surtout sa particularité par le fait qu'elle est un polar, demeure encore peu analysée. Barbero (2011), Temkeng (2007), entre-autres, ont essayé, à travers des méthodes et approches tantôt similaires tantôt divergentes, d'appréhender certains fléaux qui minent nos sociétés. Ces problèmes foisonnent dans Sorcellerie à bout portant. Ce qui n'est pas sans soulever une multitude d'interrogations: Comment apparaissent les maux sociopolitiques qui empêchent aux négro-africains du Congo de s'épanouir convenablement? Pourquoi les personnages éprouvent tant de difficultés pour bouleverser cette situation chaotique dans la laquelle ils sont confinés ? Quels procédés d'écriture mettent-ils à nu de manière feutrée les dysfonctionnements de la société congolaise ? Existe-t-il des faits assez poignants qui témoignent du réalisme de cette œuvre par le dévoilement de sa conformité aux réalités qui prévalent en RDC notamment dans le contexte socio-historique du récit?

Les thématiques de la vie sociopolitique dans les romans légitiment tout l'enjeu au tour d'elles. Les problèmes sociopolitiques qui marquent plusieurs espaces de $l^{\prime}$ Afrique subsaharienne et qui jaillissent dans plusieurs romans, expliquent l'intérêt accordé aux questions satiriques. Ce qui accorde une grande importance à l'approche sociale du texte littéraire dans cette réflexion. L'un des aspects les plus saillants dans Sorcellerie à bout portant c'est l'interpellation d'autres formes d'écriture, la cassure des frontières entre texte romanesque et genres tiers permettant une subversion de l'impérialisme générique qui chercherait à cloisonner le roman. Ainsi pour Bakhtine (1978) :

Le roman permet d'introduire dans son entité toutes espèces de genres tant littéraires (nouvelles, poésie, théâtre, ...) qu'extralittéraires (études de mœurs, textes rhétoriques, scientifiques, religieux etc....). En principe, $n^{\prime}$ importe quel genre peut s'introduire dans la structure d'un roman, et il n'est guère facile de découvrir un seul genre qui n'ait pas été, un jour ou l'autre, incorporé par un auteur ou un autre. (p.11)

Ce qui montre la grande liberté que peuvent prendre les écrivains à l'égard du genre romanesque. Ngoye ne s'en est pas privé. Il a su s'en servir pour nous présenter un texte que Bessière (1988), pourrait qualifier de «fiction hybride» du fait de sa grande richesse en faits sociaux.

Dans cet ouvrage, la corruption reste un mal qui engendre de graves dysfonctionnements dans les institutions juridiques. Quant au poids du surnaturel dans l'univers du récit, il contribue largement à entretenir la sorcellerie qui devient une pratique généralisée dans l'œuvre de Ngoye. Notre réflexion sera aussi axée sur les problématiques de la dégradation des mœurs et de l'usage des stupéfiants, véritables refuges pour s'évader des écueils qui rendent insupportables l'existence de certains personnages.

\section{MÉTHODE}

Le discours satirique de Ngoye étale les affres et les ridicules de ses contemporains tant de manière ironique qu'explicite en étant à la fois mordant et amusant ou excessif et mesuré. Ce contraste est à l'image du paradoxe qui caractérise le Congo un espace au sous-sol très riche alors que sa population vit une misère indescriptible. La sociocritique, approche analytique de cette étude, est incontournable dans l'explication des faits sociaux autant que la narratologie l'est pour les techniques 
narratives. C'est la méthode la mieux indiquée pour aboutir aux résultats escomptés. Elle nous permettra de «surfer» dans le cosmos social diffus dans l'œuvre. Ce qui est rendu possible par la dimension hybride du texte englobant en son sein des aspects littéraires et extra-littéraires notamment divers contenus sociaux. C'est ce que Duchet (1971) résume par une poétique de la socialité inséparable d'une lecture idéologique dans sa spécificité textuelle.

Elle s'intéresse aux interactions entre texte et contexte. Le premier étant un fruit du second qu'il supporte et véhicule grâce à la magie du récit. Les œuvres littéraires ont souvent été modelées par les époques qui les ont vues naitre. L'Antiquité avait sa rayonnante tragédie accordant une place privilégiée à la mythologie grecque; le Moyen âge est témoin de la création du roman de chevalerie exposant les quêtes héroïques et les duels d'honneur dans une France rustique plus moralisante que morale ; les années 1930 furent marquées par la poésie de la Négritude s'opposant à la volonté de «néantisation » du Noir victime de la colonisation. Aussi le Congo sous l'emprise de la dictature a aussi sa littérature avec des auteurs comme Sony Labou Tansi ou Achile Ngoye.

Ethiopiques revue de littérature et de philosophie, créée en 1975 par Léopold Sédar Senghor, publiant deux fois par an et bien d'autres revues de haute renommée publient depuis des décennies des articles s'appuyant sur cette approche sociologique comme méthode de recherche et d'analyse du texte littéraire. $\mathrm{Du}$ fait que la réalité sociale façonne largement les œuvres produites reflétant la société, les modes de vie des peuples, leurs aspirations, leur joie et leur souffrance. C'est ce que Mukařovský (1936) appelle «la conscience collective». Pour Tamine et Hubert (2002), la sociocritique apparait comme une tentative pour expliquer la production, la structure et le fonctionnement du texte littéraire par le contexte politico-social.

Nous procéderons par alternance en prenant en charge dans chaque phase les manifestations sociales qui caractérisent les fléaux à étudier en les appuyant par des faits concrets bien référés ainsi que des commentaires détaillés à valeur de discussion pour mieux les cerner.

\section{RÉSULTATS ET DISCUSSION}

\section{La Sorcellerie}

L'émergence de l'Afrique dans la société de consommation a donné lieu à des comportements aussi avilissants les uns que les autres. Parmi ceux-ci, la sorcellerie, pratique très ancienne, et la corruption qui à côté de l'impunité occupent une place de premier rang.

Chez Ngoye la sorcellerie occupe une grande place. En effet, les personnages de premier plan sont impliqués dans le fétichisme. Ils $s^{\prime} y$ intéressent soit pour se protéger soit pour nuire à autrui soit pour accéder à des avantages, par exemple pour bénéficier d'une promotion dans leur carrière. Cette pratique mystique qui réunit à la fois des éléments du christianisme et de l'animisme, était très fréquente dans les sociétés négro-africaines encore sous influence de la tradition. Elle est rangée dans la catégorie de la sorcellerie qui est plutôt une démarche individuelle qu'une action collective menée par un groupe. Elle est observée en solitude dans un isolement total loin des regards indiscret et de certaines langues.

La sorcellerie est en ce sens moins acceptée que les autres pratiques spirituelles. Elle est souvent dénoncée comme une démarche anticommunautaire pour ne pas dire égoïste de la part de celui qui la pratique et destructrice dans la mesure où certains ne se privent pas de l'utiliser pour maîtriser ou éliminer un adversaire ou un rival dans le domaine professionnel ou sentimental.

La présence de ces pratiques s'explique notamment par le fait que l'Afrique a connu, pratiquement, pendant des millénaires une seule religion qui est l'animisme avec toutes les pratiques qui le caractérisent. C'est un héritage culturel qui apparaît dans nos textes. Il est aussi à noter que les pratiques animistes vont, 
avec l'arrivée du christianisme, perdre progressivement leur importance et leur originalité. Le syncrétisme religieux y est fortement présent. On assiste à un mélange des deux sphères religieuses d'où la présence de la croix, des bougies dans les lieux de culte. Par ailleurs, on ne peut pas parler de sorcellerie sans parler du sorcier qui est au début et à la fin de toute pratique de ce genre. Voici comment Bureau (2002) parle du sorcier, à la fois coupable et victime :

La croyance généralisée aux sorciers mangeurs d'hommes combine l'aspect actif et l'aspect passif du meurtre rituel. Le sorcier, homme ou femme de la nuit, clandestin, est censé accroître son pouvoir propre en s'incorporant la substance des autres: cette opération se fait par le truchement des doubles: la personnalité a plusieurs composantes. Une fois mangée, la victime n'est plus visible que sous l'apparence de son ombre. Mais le groupe social est à l'affût de ceux qui se sont ainsi mis hors la loi et bénéficient de pouvoirs surhumains au détriment du commun. Si des inégalités apparaissent dans la communauté, si la maladie et la mort sont plus fréquentes que la normale, les individus dominants ou marginaux sont suspectés et, par divers procédés (dont l'ordalie est le plus courant) convaincus de sorcellerie et éliminés physiquement. (p.37)

Le sorcier pouvait, autrefois avant l'arrivée des religions révélées (l'Islam et le christianisme), aller jusqu'à demander des sacrifices humains. Ce genre de pratique était très fréquent. Ces sacrifices vont progressivement reculer jusqu'à être bannis totalement par les sociétés qui vont subir l'influence des nouvelles croyances révélées qui, dans leurs livres, vont accorder une bonne place au caractère sacré de la vie humaine qui ne peut être ôtée pour aucune pratique spirituelle.

Cependant, la soif du pouvoir, de l'avoir ou du meilleur être peut pousser certains individus sans scrupule à verser du sang humain pour arriver à leur fin. Cette réalité qui relève à la limite du cynisme pour ne pas dire de la barbarie est bien présentée dans l'œuvre de Ngoye :
Les sacrifices humains sont indispensables de nos jours. Parce qu'ils s'effectuent dans le plus grand secret et qu'ils relèvent de l'hermétisme. Marginalisés par l'évolution de la société, les sacrificateurs, qui ne sont rien d'autre que les sorciers, ces empoisonneurs de notre milieu ne charcutent pas forcement (...) la menace qu'on découvre à poil au grand matin, perchée sur un arbre et qui s'avère y nicher à perpète. (Dozon, 2008, p.138)

Ngoye à travers le narrateur dénonce cette pratique d'une autre époque qui perdure encore dans certaines sociétés. L'auteur évoque surtout son caractère secret. Cela s'explique notamment par l'évolution de nos sociétés et par le fait que prendre la vie d'autrui, en dehors de toute sentence judiciaire, est considéré comme un crime lourdement puni par la loi.

La survie des sacrifices humains peut s'expliquer, au-delà de ces origines culturelles, par la pauvreté qui gangrène les sociétés négro-africaines. Aussi pour, soidisant, prétendre à de meilleures conditions de vie, accéder à plus de réussite socioéconomique ou pour conserver leurs privilèges, certains organisent-ils et entretiennent-ils secrètement de telles pratiques. Les victimes sont généralement issues des classes les plus démunies de la société notamment des marginaux. Comme partout ailleurs, les pauvres et les faibles sont toujours les plus exposés aux dangers de toutes sortes.

«Autrefois ces sacrifices se faisaient de manière solennelle en plein jour " (Ngoye, 1998, p.116). Car ils étaient considérés comme normaux; mieux, comme indispensable à l'équilibre de la communauté à la préservation de la paix, de la prospérité, et du bien-être des membres de la société. $C^{\prime}$ est dire que c'était une pratique qui permettait d'éloigner les mauvais sorts et d'attirer des bienfaits. Mais Ngoye nous expose des pratiques qui se font la nuit et dans la plus grande discrétion. Par ailleurs, il est à noter qu'elles ont perdu leur caractère collectif et ne visent plus les intérêts de la communauté mais ceux égoïstes d'un individu ou d'un petit groupe ayant les mêmes intérêts. 


\section{La corruption}

Elle est présente au sein de toutes les couches sociales, elle semble essentiellement être le fait des élites politiques, des cadres de l'administration publique, des « hommes de tenues ». Généralisée dans tous les domaines, la corruption s'est amplifiée dans certains milieux au point qu'elle est devenue une véritable règle de vie au grand dam des populations, notamment les plus défavorisées, c'est-à-dire les pauvres qui se voient mépriser dans les services que l'on devait les rendre sans bourse donner en contrepartie.

Sans doute l'un des plus grands maux de la société africaine moderne, la corruption est vue par de nombreux romanciers africains comme un fléau des temps modernes. Même si l'histoire des sociétés africaines traditionnelles nous apprend que c'est une pratique qui est constatée timidement jadis, notons qu'elle s'est aggravée avec la modernité.

Ce phénomène est dû à la combinaison de plusieurs facteurs, le plus important étant l'impunité généralisée que nous aborderons plus tard. La notion de service ou de bien public est reléguée au second plan. Elle a perdu tout son sens dans beaucoup de pays $\mathrm{du}$ continent noir, ce qui donne lieu à des pratiques comme la corruption. Le lecteur du corpus est séduit par la manière dont ce fléau est présenté. Les auteurs nous montrent que tout peut s'acheter dans cette société. Il est même possible de bénéficier d'une promotion au sein d'un corps si l'on a de bonnes relations ou si l'on est assez aisé.

La corruption traverse tout le récit, c'est une pratique généralisée dans toutes les institutions au niveau de tous les services. Dès le début du récit l'auteur nous en montre une de ses facettes à travers un douanier, personnage travaillant à l'aéroport de Kinshasa :

L'œil exercé du douanier dénicha une enveloppe épaisse, la palpa en guignant le pigeon. (...) « Tu as vraiment du bol, lui susurra-t-il en piochant sur les 10 000FF les obsèques. Bicause je peux te coffrer pour l'importation frauduleuse $\mathrm{d}$ 'une monnaie étrangère: un crime économique passible d'un long séjour au gnouffe. Mais on est cools, puisqu' on se partage moite-moite, solidarité oblige. Le requin empocha quelques billets, en refila d'autres à ses collègues. (Ngoye, 1998, p.20).

Le romancier congolais, nous montre la généralisation de la corruption et sa banalisation par les services étatiques, notamment par la douane. Les voyageurs qui transitent par l'aéroport sont particulièrement victimes de ce fléau qui gangrène la société négro-africaine moderne. Son ampleur est telle que ceux qui la pratiquent ne se gênent pas devant leurs collègues ou victimes. Ils agissent sans scrupule en complicité avec des collègues ou d'autres individus véreux. La corruption n'épargne personne et peut amener le plus intègre des citoyens à céder à la tentation du gain facile.

Ce fléau qui nuit à la justice dans de nombreux pays africains, est rendu possible chez Ngoye, notamment, par le fait que les commanditaires du meurtre du Major Tsham sont d'influents cadres supérieurs de l'armée congolaise. Ils peuvent ainsi user de leurs situations pour faire pressions sur les enquêteurs ou les juges. Ces derniers sont aussi corrompus que mal formés.

Dans une pareille situation l'option de l'auteur colle bien avec la réalité. Si l'on sait aussi que la dictature qui prévalait dans ce pays (le Zaïre sous le règne du Président Mobutu) protégeait les militaires qui étaient investis de beaucoup de pouvoirs. Ce qui permet au tyran de s'éterniser à la tête du pays aussi longtemps que les agents de la force publique y trouvent leur compte.

Mais la presse joue de plus en en plus un rôle déterminant dans la lutte contre ce fléau. Aussi, malgré l'existence de cercles d'influence formés, notamment par des personnalités de l'Etat qu'elles soient politiques, militaires ou diplomatiques, les médias demeurent-elles relativement indépendantes dans de nombreux pays d'Afrique noire. Elle est même considérée comme un quatrième pouvoir à côté du législatif, de l'exécutif et du judiciaire. C'est ce que Kizito a compris. Aussi, sachant le manque de justice dans son pays, va-t-il faire appel à la radio pour une diffusion des conclusions de l'enquête sur la mort de son frère : 
Nouvelles de dernière minute: le Courrier d'Afrique publie, dans sa livraison de ce matin, une enquête sur le meurtre, déguisé en accident de roulage, d'un dirigeant de la milice. L'auteur présumé de cet assassinat est un ancien officier des FAS. Des éléments de la Gendarmerie cernent en ce moment son domicile du quartier vitamines II où, selon le quotidien qui cite une source sûre, un autre corps dont la mort remonte à plusieurs jours, est enfermé dans une chambre. (Ngoye, 1998, p.22).

Dans une procédure judiciaire, après l'élucidation d'un crime, le dossier, c'est-àdire les résultats de l'enquête, est remis à la justice pour un jugement. Mais dans ce récit, l'auteur a voulu respecter la logique qui aimerait que dans un pays où le système judiciaire est à genoux, la seule possibilité qui s'offrait à Kizito c'était de prendre à témoin les opinions nationale et internationale sur cette affaire, à travers la Presse qui est ici une véritable alternative face à la défaillance du système judicaire.

Si l'ex-capitaine, un des commanditaires du meurtre du Major est arrêté, force est de savoir que l'impunité est toujours présente dans cette œuvre. Dans la mesure où les autres officiers qui sont impliqués dans ce crime ne sont pas mis aux arrêts du fait de leur situation d'officiers en activité pouvant donc influencer toute décision judiciaire. Ces agents des forces de l'ordre sont considérés par Bébé (2006) comme des privilégiés d'un système fermés et sont ainsi des intouchables tant qu'ils disposent de marges de manœuvres offertes par le pouvoir.

Ngoye aborde le rôle de certains personnages qui, de par leurs fonctions assez élevées, peuvent entraver toute action de justice et entretenir ainsi l'impunité qui, au même titre que la corruption, donne une image négative des institutions étatiques notamment juridiques.

\section{L'espace urbain: un terreau de criminalité}

L'espace urbain comme Kinshasa capitale de la République démocratique du Congo chez Ngoye est une véritable source de violence. Il s'oppose à la campagne qui, du fait de son conservatisme, de son hostilité vis-à-vis de tout ce qui est étranger à ses traditions, à son mode de vie, à sa religion, rejette la modernité, mal comprise, qu'incarnent les grandes villes. Même s'il y a interaction, entre les milieux urbain et rural, rendue possible par les activités des personnages qui y évoluent, la campagne se présente comme un milieu en métamorphose alors que la ville montre le visage d'une modernité très problématique, mère de tant de souffrance.

La ville est mal vue car c'est le lieu où toutes les vices, toutes les dérives sont observées. C'est un milieu «monstrueux» qui dévore toute attitude conforme au modèle traditionnel, toute valeur positive et installe les attitudes les plus haïssables, les tares les plus repoussantes: corruptions mensonge, égoïsme, individualisme voire même le cynisme de certains de ses habitants. Autant de choses que condamnent les vieux sages des villages au point qu'ils sentiront la nécessité de dénoncer le comportement d'individus qui, jadis, étaient considérés comme des références quand ils évoluaient chez eux, c'est-à-dire dans l'espace rural.

L'espace urbain apparait comme une entité chargée négativement comme le lieu dans lequel se déroulent toutes les crises notées où se constatent leurs causes justifiant dans une certaine mesure le mobile de certains crimes dans l'œuvre de Ngoye. Sa représentation sous cette apparence n'est pas une spécificité de Sorcellerie à bout portant. Elle est visible dans plusieurs autres textes de la riche littérature négro-africaine contemporaine. Ce qui peut avoir plusieurs significations. La ville est un facteur qui participe au changement de comportement dans le sens de la dégradation des mœurs des ruraux qui sont souvent caricaturés comme des personnages naïfs ou manipulables par des citadins sans scrupule. À ce niveau, cet espace est conçu comme une machine à pervertir, à faire adopter de nouvelles attitudes et manières de vivres en déphasage avec les mœurs d'autrefois. 


\section{La dégradation des mœurs}

Cette thématique est très fréquente dans la littérature négro-africaine notamment celle de la troisième période dénonçant certains faits socio-politiques et autres pratiques constatées en Afrique. L'auteur, à travers la voix des différents personnages (enquêteurs, prostituées, barmans) expose le visage d'une société qui perd progression certaines de ses valeurs fondamentales comme la pudeur, la fidélité, l'endurance dans la misère, l'abstinence, le respect du prochain.

Cette perversion ne se voit pas seulement chez les jeunes. Les personnages les plus âgés également peuvent être concernés. En effet, dans Sorcellerie à bout portant, Maisha, épouse de la victime, ne s'est pas privée de chercher à coucher avec Kizito, alors qu'elle était en veuvage :

$\mathrm{Tu}$ sais bien que cela est interdit entre nous, Seneki. C'est tabou, inadmissible. La veuve souleva la tête au bout d'un moment, les billes embuées de larmes. Elle cherche le regard insaisissable. Mais son beau-frère, persuadé qu'une grillade, aussi furtive soit-elle, faisait le lit de l'inceste, l'évita comme la peste, Gelé un peu plus par le contact physique, il repoussa la charmeuse non sans cogiter sur le meilleur moyen de se dépêtrer du piège (Ngoye, 1998, p.220).

Le caractère sacré du veuvage est ici violé par Maisha. Cette dernière est présentée à l'image de toutes les femmes qui, ayant perdu leurs époux, ne se gênent pas à sortir avec d'autres. Pour Zahan (1970), La tradition négro-africaine est claire en ce qui a trait aux évènements qui marquent la vie de l'homme en général et particulièrement concernant le deuil. La femme du défunt doit se retirer de toute activité mondaine, elle doit manifester sa douleur causée par la perte d'un être cher avec qui elle a eu à vivre des moments importants. Le rejet de ce principe social par Maisha est un moyen pour l'auteur de montrer le mépris des traditions que l'on constate de plus en plus dans nos villes et la primauté du plaisir sur les valeurs sociales traditionnelles.

La dégradation des mœurs touche principalement les jeunes qui ont souvent une vie sexuelle libérée qui s'apparente le plus au libertinage. Ils ne semblent pas être soumis à aucune morale ni limite encore moins logique si ce n'est celle de la recherche du plaisir.

L'alcool est la drogue la plus présente dans le corpus contrairement à ce que l'on constate dans beaucoup de romans noirs occidentaux notamment ceux qui sont écrits par des auteurs américains. Ces derniers nous présentent des récits dans lesquels les autres drogues comme l'héroïne et la cocaïne apparaissent plus fréquemment. Elles sont souvent à l'origine de la violence dans les banlieues et de la forte criminalité d'une société victime d'un développement à deux visages qui opposent d'une part les quartiers luxueux où résident les plus riches et d'autres part les ghettos où «nichent» les pauvres sombrant généralement dans la drogue. D'ailleurs, c'est cette situation d'inégalité sociale et de misère qui va jeter les bases du roman noir aux Etats-Unis avec une multiplication des meurtres et des toxicomanes.

Cela n'est pas le cas chez Ngoye où l'alcool est la drogue la plus présente. L'absence des autres types de drogue peut s'expliquer par le réalisme de nos auteurs. En effet, dans les sociétés négro-africaines même modernes, il est très difficile de voir des gens s'alarmer sur l'ampleur de la présence de stupéfiants qualifiés de drogues dures comme on en voit dans les pays développés. Cela s'explique par la cherté de ces produits nuisibles qui les place hors de portée des populations des pays sous-développés.

Le thème de l'alcoolisme est abordé sous plusieurs angles. Même s'il est la boisson favorite pour faire la fête où pour créer une ambiance de jouissance, l'auteur rappelle au lecteur les effets nocifs de l'alcool sur la physiologie humaine. Il est considéré comme un fléau qui empêche à l'individu d'être maître de lui-même. En perdant toute lucidité, il peut se mettre à commettre des actes répréhensibles, rejetés par la société car non-conformes à 
la loi encore moins aux valeurs traditionnelles ou religieuses :

Pour toute réponse, Maicha dégagea sa louche gardée sous le pagne, exhibant une boutanche de scotch coiffée d'un verre. Précision immédiate: le whisky provenait des achats effectués lors de la dispersion du deuil public. Elle le décapsula, servit le soiffard encore plus soul. Examen rapide du décor. Elle vient enfin au beau-frère toujours bala, relança la causette. (Ngoye, 1998, p.98).

Après la prise de l'alcool, la femme du défunt en veuvage se met à faire des avances au frère du Major qui fut son mari. Ce qui constitue une violation terrible du caractère sacré du deuil. Le narrateur fait précéder cette situation dégradante par la mise en scène des deux personnages en train de prendre un whisky, l'auteur expose l'un des effets de l'alcool. En ce sens qu'il nous plonge dans un autre univers dans lequel l'individu, écarté de la réalité, se permet de transgresser les règles les plus élémentaires de la société. $C^{\prime}$ est donc une sorte de mise en garde pour le lecteur au-delà du fait que l'alcool nous fait perdre la maîtrise de nous-mêmes. Comme le montre Achille Ngoye, il est un produit qui agit sur l'organisme et transforme l'individu en lui donnant une autre apparence (Salmandjee, 2003).

Ngoye ne s'est pas privé de se servir de certains procédés satiriques comme l'ironie, l'antiphrase, la caricature, l'euphémisme, le paradoxe ou l'oxymore. Tout en entretenant une communion entre le lecteur et le romancier. Ces outils de rhétorique permettent à l'écrivain de s'attaquer, avec intelligence et gaieté, aux tares de sa société sans paraître désagréable ni se mettre en péril face à la censure, aux menaces de mort, de déchéance sociale et autres dangers liés aux régimes et autoritaires.

\section{CONCLUSION}

La satire sociale dans Sorcellerie à bout portant se matérialise ainsi à travers les pratiques occultes, la corruption, l'impunité, la dégradation des mœurs qui sont autant de maux qui minent beaucoup de sociétés africaines modernes. Ce discours dénonciatif n'épargne aucune couche sociale, aucun âge.
Tous les espaces sont interpellés aussi bien la campagne que la ville, même si cette dernière est la plus touchée.

Suivant une approche qui mêle réalisme langagier et connaissance profonde de son pays d'origine, Ngoye a su exposer ce que le romancier camerounais Mongo (2009), appelle «les plaies $d u$ continent noir ». Le texte est très poignant par la diction mais surtout par la manière dont la fiction et le réel se manifestent dans le roman qui apparaît, sans doute, comme un miroir qui montre sans complaisance un certain malaise social. Ce qui en fait, du coup, un véritable instrument de connaissance de la société congolaise contemporaine.

Cette peinture objective n'est pas l'expression d'une vision pessimiste sur le devenir de l'Afrique noire. Mais elle peut être comprise comme l'extériorisation d'un ras-le-bol, d'une révolte face à une situation proche du chaos. Ce qui traduit un désir profond de changement chez l'auteur qui cherche, par cette écriture satirique, un remède possible au mal social à l'instar de beaucoup d'autres romanciers négroafricains.

\section{REMERCIEMENTS}

La réalisation de cet article a été possible grâce au concours de plusieurs personnes à qui je voudrais témoigner toute ma reconnaissance : Le Professeur Amadou LY Directeur de recherche à l'Université Cheikh Anta Diop de Dakar pour tous ses conseils, Le responsable de la bibliothèque départementale mais aussi toute l'équipe de la revue FRANCISOLA qui m'a permis par les références envoyées de me corriger et d'adapter mon travail aux critères recherchés.

\section{RÉFÉRENCES}

Bakhtine, M. (1978). Esthétique et théorie du roman (traduit du russe par Daria Olivier, préface de Michel Aucouturier). Paris : Gallimard.

Barbero, O. (2011). Peut-on représenter le mal ? La rédemption et le mal. Repéré à 
http://www.revue-alkemie.com/008alkemie-le-mal.html.

Bébé, B. E. (2006). Presse écrite et expériences démocratique au Congo-Zä̈re. Paris : Harmattan.

Bessière, J. (dir.). (1988). Hybrides romanesques : fiction (1960-1985). Presses universitaires de France.

Bureau, R. (2002). Anthropologie, religions africaines et christianisme. Karthala Editions.

Dozon, J.P. (2008). L'Afrique à Dieu et à Diable : États, ethnies et religions. Paris : Ellipses.

Duchet, C. (1971). Pour une socio-critique, ou variations sur un incipit. Paris : Armand Colin.

Mongo, P. (2009). Congo-Kinshasa : La nouvelle génération d'écrivains négro-africains. Repéré http://www.lepotentielonline.com.
Mukařovský, J. (1936). Estetická funkce, norma a hodnota jako sociální fakty $=$ : Fonction, norme et valeur esthétiques comme faits sociaux. Praha: F. Borový.

Ngoye, A. (1998). Sorcellerie à bout portant. Paris : Gallimard.

Salmandjee, Y. (2003). Les drogues, Tout savoir sur leurs effets, leurs risques et la législation. Paris : Eyrolles.

Temkeng, A.E. (2007). Sémiotique du chaos et folie dans le roman camerounais : Temps de chien de Patrice. Repéré à http://ethiopiques.refer.sn/spip.php?pa ge=imprimer-article\&id_article=1542.

Tamine, J. et Hubert, M. C. (2002). Dictionnaire de critique littéraire. Armand Colin.

Zahan, D. (1970). Religion, spiritualité et pensée africaines (Vol. 374). Payot. 